\title{
LÓGICAS NORMATIVAS, MORAL Y DERECHO
}

\author{
LEILA Z. PUGA
}

Pontificia Universidade Católica de São Paulo

NEWTON C. A. DA COSTA

Universidade de Sāo Paulo

ROBERTO J. VERNENGO

Universidad de Buenos Aires

\begin{abstract}
El matemático tendrá que tomar en cuenta no sólo las teorías que se acercan a la realidad, sino también, como en la geometría, todas las que son lógicamente posibles. Siempre tiene que estar atento para lograr una visión completa de todas las conclusiones derivables de los sistemas de axiomas de partida.

D. Hilbert, Mathematical Problems, 1900.
\end{abstract}

\section{Introduccion}

La logica deóntica puede ser encarada de dos maneras, a saber: una descriptiva y otra prescriptiva, según la interpretación que sè atribuya a los enunciados normativos. La distinción entre enunciados normativos con sentido descriptivo y enunciados normativos con sentido prescriptivo se remonta, en la teorfa del derecho, a la distinción entre Rechtssatz y Rechtsnorm, que Hans Kelsen utiliź y analizó hace ya varias décadas. La distinción reaparece en filósofos posteriores, como G.H. von Wright, entre otros, quienes diferencian usos descriptivos y usos prescriptivos de las oraciones normativas. La lógica de las normas - llamada genéricamente "lógica débntica" a partir del ensayo de von Wright (1951) "Deontic Logic"- puede 
ser vista como una lógica de formulaciones normativas formalizadas, interpretadas descriptivamente ("a logic of descriptively interpreted formalized norm-formulations"). Cabe discutir la conveniencia de denominar lógica deóntica a esta lógica que se ocupa de enunciados descriptivos, pues es tema polémico el aceptar una lógica aplicable a las normas en su función prescriptiva (Alchourrón, 1969; von Wright, 1983; Hilpinen, 1971 y 1981).

En este ensayo, sin perjuicio de opiniones dispares que los autores puedan sustentar, adoptaremos la primera interpretación, la descriptiva, como continuación de investigaciones anteriores (da Costa y Carnielli, 1986; Puga y da Costa, 1987a, 1987b y 1987c; Puga, 1985 y Vernengo, 1987); la misma refleja la tendencia actual dominante en el pensamiento iusfilosófico. No debe considerarse una toma de posición con respecto a las posibilidades de lógicas aplicables a las normas en función prescriptiva, ni tampoco una decisión con respecto a la cuestión terminológica con que esas lógicas son denominadas en los trabajos sobre el tema.

En su forma normal la lógica de6ntica puede ser entendida como una extensión de la lógica clásica modal, mediante la introducción de operadores deónticos específicos, $O$ (obligatorio), $P$ (permitido), $V$ (prohibido) y $F$ (facultativo o indiferente). Cabe sostener que la lógica deóntica, así entendida, se ocupa principalmente de las propiedades formales de esos operadores, así como de cuestiones correlacionadas. En una lectura superficial, tales operadores son entendidos como giros adverbiales que dan un sentido modal a los verbos principales, conforme al código normativo de que se trate. Así, tratándose de una prescripción moral, como el precepto "prohibido mentir", "prohibido" es entendido como la modalidad moral atribuida a la acción de mentir, esto es, como el deber de no mentir (si, como es tradicional en el pensamiento ético, se parte de la noción de deber, o de obligación, como término primitivo). Junto a las prohibiciones morales, en forma simi- 
lar tenemos obligaciones y permisiones jurídicas. En contextos legales —como "es obligatorio pagar impuestos"-, los operadores deónticos mencionados son referidos a contenidos jurídicos, principalmente, acciones, actos y hechos. Aquí "obligatorio pagar" quiere decir que se trata de un deber desde un punto de vista jurídico y pari passu para los restantes operadores. Por consiguiente, tendríamos una lógica resultante deóntica de carácter jurídico. Sin embargo, en los lenguajes naturales no siempre es claro el sentido que se atribuye a los términos que expresan esos operadores. Por ejemplo, el opera$\operatorname{dor} F$ (facultativo) no tiene siempre sentido prescriptivo comprensible en el discurso moral y, en consecuencia, tampoco es claro su eventual sentido moral descriptivo. De ahí que algunos éticos no consideren asunto moral la consideración de las acciones indiferentes o adiáforas.

En la tradición filosófica relacionada con el derecho y la moral, o sea, con el lenguaje ético en sentido lato, la distinción entre normas morales y normas jurídicas (en sentido prescriptivo) se efectúa por lo general a partir de los contenidos de los enunciados y no a partir de las modalizaciones deónticas utilizadas. Vale decir, se piensa que los enunciados o proposiciones aléticas, afectadas luego por los operadores deónticos, se refieren a actos morales o a actos jurídicos. Esta concepción deriva de la creencia filosófica en la existencia de hechos morales o jurídicos a los que corresponderían los respectivos enunciados normativos, tanto en su interpretación prescriptiva como en su versión descriptiva. Cabe también sostener, conforme a una tradición clásica, que los modalizadores, aléticos y deónticos, reflejan actitudes de creencia con respecto a los enunciados. Tal es la interpretación clásica de las modalidades de los juicios, como en Kant o en Husserl. Por consiguiente, la distinción entre proposiciones morales o jurídicas, nunca es puramente formal en esas formas tradicionales de pensamiento. Pero cabe también pensar las modalidades deónticas como rasgos gramaticales que expresan modalidades decisionales; esto 
es, actitudes volitivas atribuidas a las referencias de las oraciones. Ésta, por ejemplo, es la interpretación que seguramente Kelsen propuso. Esta interpretación volitiva conlleva también una interpretacion no puramente formal de los operadores deónticos. En los trabajos anteriores de los autores, en especial los de Puga y da Costa, se intentó algo así como una interpretación de los propios operadores deónticos no exclusivamente formal, como en las logicas normativas clásicas, sino una interpretación, por decirlo asi, relativamente formal, en la cual los operadores referidos, y no s6́lo los enunciados descriptivos modalizados, poseen un rasgo o contenido ético, sea moral o jurídico.

La relación fundamental entre esos dos tipos de obligación, la obligatoriedad moral $\left(O_{m} A\right.$ simboliza que el acto $A$ es moralmente obligatorio) y la obligatoriedad jurídica $\left(O_{j} A\right.$, por su parte, significa que el acto $A$ es jurídicamente obligatorio, donde $A$ puede entenderse también como la oración o proposición que describe un hecho o un acto). En los primeros trabajos mencionados de Puga y da Costa, se definieron las siguientes relaciones: lo que es jurídicamente obligatorio es moralmente permitido (donde $P_{m} A$ simboliza que el acto $A$ está moralmente permitido); y lo que es moralmente obligatorio no puede estar jurídicamente prohibido (donde $V_{j} A$ significa que el acto $A$ está jurídicamente prohibido). Estas dos relaciones fueron representadas simbolicamente por los dos siguientes axiomas o principios:

$$
O_{j} A \rightarrow P_{m} A
$$

y

$$
O_{m} A \rightarrow P_{j} A,
$$

donde el símbolo “ $\rightarrow$ " representa la implicación material.

En dichos trabajos la moral (o el discurso ético latu sensu) y el derecho se encuentran íntimamente correlacionados. En 
Puga y da Costa (1987a), inclusive, se estudia un sistema $D$ de lógica déntica, con modalidades morales y jurídicas, en el que esas modalidades se encuentran ligadas por ciertos principios específicos que se enumerarán en la siguiente sección. El sistema $D$ fue creado con el objetivo de intentar una mejor comprensión de las conexiones recíprocas entre dos tipos de obligaciones, ensayo que puede ser de gran importancia para el análisis de ciertas teorías iusfilosóficas.

En efecto, es cosa sabida que, tanto en el pensamiento tradicional como en el actual de la filosofía del derecho, así como en los presupuestos de muchas ideologías morales y religiosas, la cuestión de las relaciones entre normas o principios morales y preceptos jurídicos tiene soluciones varias. Para el positivismo jurídico más radical, cuyos representantes clásicos son considerados Austin y Kelsen, no habría relaciones funcionales entre normas jurídicas y morales: la moral, de este modo, estaría separada del derecho, como ya Thomasius lo proclamara hace siglos. Para muchas tendencias filosoficas iusnaturalistas, en cambio, el derecho positivo está estrechamente relacionado con la moral, o, como suele decirse, con el derecho natural, sea porque se considera al primero derivado deductivamente de la moral, o, por lo menos, incluido en ella por una relación de subalternación ( $c f r$. Vernengo, 1989). En rigor, se trata de postular o definir relaciones posibles entre conjuntos de enunciados normativos; las relaciones definibles son muchas. En la teoría contemporánea del derecho la cuestión aparece, por lo común, como el problema de las relaciones entre normas jurídicas positivas y sistemas de valores morales, individuales o sociales, reales o ideales. Algunos pensadores estiman que el conocimiento que la ciencia jurídica actual alcanza es parcial, en la medida en que sólo toma en cuenta los elementos normativos del derecho sin atender lo suficiente a los datos valorativos. Paralelamente, existen tendencias que destacan que la ciencia jurídica no recoge de manera apropiada los elementos fácticos (sociológicos) que los datos jurídicos exhibirían. 
En la tradición más rígida del positivismo jurídico se propone que el conocimiento científico del derecho se limite a la descripción, explicación y sistematización de las normas jurídicas positivas, excluyendo enteramente datos empíricos de índole sociológica o datos valorativos como las pautas morales. Las ciencias jurídicas existentes adoptan, a este respecto, posiciones eclécticas. Por cierto que, tanto en la teoría jurídica como en el pensamiento iusfilosófico aparecen propuestas para elaborar un conocimiento científico del derecho abierto a otras dimensiones que las puramente normativas. En nuestros países, por ejemplo, cabe apuntar las sugerencias de C. Cossio en la Argentina y, en Brasil, las de M. Reale, para quienes el derecho aparece como un fenómeno pluridimensional ( $c f r$. Cossio, 1945; Reale, 1986 passim). Estas teorías, en todo caso, aparecen como sumamente complejas desde un punto de vista lógico, puesto que, de aceptarlas como teorías admisibles, sería menester estructurar sistemas lógicos aptos para hacerse cargo de todas las dimensiones postuladas. Puga y da Costa (inicialmente en 1987b) han indagado acerca de algunos de los presupuestos lógicos posibles de las concepciones tridimensionales del derecho, como la de M. Reale, según la cual el derecho exhibe siempre dimensiones fácticas, normativas y axiológicas, intentando una formalización inicial de algunos aspectos de esas teorías. Vernengo, desde un punto de vista más general, ha discutido la consistencia interna de algunas de las teorías que ven en el derecho un fenómeno con múltiples aspectos categorialmente diferentes (Vernengo, 1990).

Cabe agregar, como ha resultado de varias experiencias destinadas a construir sistemas expertos en derecho o permitir un análisis automático de enunciados jurídicos, que para abarcar en forma más refinada, no sólo la concepción del derecho de estas teorías pluridimensionales, sino teorías más finas de los datos jurídicos, sería preciso también ampliar los sistemas deónticos, como el denominado $D$, por lo menos con una lógica de la acción que permita enunciar normas condicionadas por 
circunstancias fácticas, así como expresar relaciones temporales. Ello ya se encuentra en el temprano ensayo de G. von Wright de 1963, Norm and Action, y ha sido reiterado recientemente por L. Allen y Ch. Saxon (1984). El tipo mismo de lógica que funcionaría como sistema de derivación deductiva admitiría diversas opciones, entre las cuales se han sugerido lógicas relevantes, lógicas naturales y otras (Miró Quesada, 1988; Alchourrón y Martino, 1989). Fuera de los desarrollos que abajo se efectúan en ese terreno, consideramos que, si han de tomarse en cuenta los aspectos valorativos del derecho, se hace preciso introducir en el sistema lógico desarrollado alguna relación de orden o de preferencia que sirva para escoger y decidir entre los valores en juego, sean ellos intrínsecos o extrínsecos al derecho mismo.

Resulta que un análisis no formal del significado de las modalidades, tanto morales como jurídicas, del sistema $D$, ha mostrado que sus postulados implican algunas consecuencias contraintuitivas (Vernengo, 1989). Pareciera, pues, que la construcción de sistemas lógicos deónticos bidimensionales —entendiendo por tales los que involucran conceptos morales y jurídicos- presenta ciertas dificultades. Por ello, es nuestro propósito, en este trabajo, continuar la investigación formal de las interrelaciones entre moral y derecho, proponiendo otras maneras de correlacionar lógicamente los conceptos morales y jurídicos. Por consiguiente, exponemos a continuación diversos sistemas posibles de lógica deóntica destinados a llevar adelante ese objetivo. Quede claro que cada uno de los sistemas lógicos propuestos define un sistema conceptual jurídico-moral; compete al filósofo, al moralista y al jurista escoger el que mejor sirva a sus propósitos específicos. Téricamente, estas propuestas pueden tener interés en la medida en que buena parte de los moralistas y juristas que trabajan científicamente en el terreno de la ética, si bien asumen que las tesis que explícitamente sustentan tienen consecuencias racionalmente derivables, suelen pasar por alto la circunstancia importantísi- 
ma de que esos conjuntos de consecuencias pueden diferir en forma considerable según sea el sistema lógico que se presuponga. Hacer más explícitos los sistemas lógicos posibles que el pensamiento ético puede adoptar es una contribución al trabajo de crítica racional del derecho y de la moral.

\section{Algunos sistemas deónticos bidimensionales}

Dada la naturaleza de este artículo, creemos conveniente hacer un resumen inicial algo detallado de los resultados principales obtenidos en relación con el sistema $D$, que constituyó nuestro punto de partida.

\subsection{Descripción del sistema $D$}

Partiendo de lo expuesto en Puga y da Costa (1987a) y Vernengo (1989), supongamos que todo lo jurídico debe ser también moral y que lo moralmente obligatorio debe estar jurídicamente permitido. Esos principios quedan simbolizados en los siguientes esquemas:

$$
O_{j} A \rightarrow O_{m} A
$$

y

$$
O_{m} A \rightarrow P_{\mathrm{j}} A
$$

En $D$, pues, son dos los axiomas (o postulados mixtos) que expresan las relaciones entre los dos tipos de modalidades: la obligación moral y la obligación jurídica. Estos axiomas, junto a los restantes postulados, permiten construir el sistema $D$. Este sistema retrata, entonces, un sistema jurídico que se encuentra relacionado, en una forma idealizada, con la moral (sea ésta la que fuere).

El sistema $D$ queda formalizado como sigue: los símbolos primitivos de $D$ son 1 ) los conectivos $\rightarrow$ (implicación), ᄀ (negación), $O_{m}$ (moralmente obligatorio) y $O_{j}$ (jurídicamente 
obligatorio); 2) un conjunto de variables proposicionales; y $3)$ paréntesis. La equivalencia, $\rightarrow$, la conjunción, $\wedge$, la disyunción, $\vee$, así como los restantes operadores morales y jurídi$\cos$, y la noción de fórmula, se introducen de la manera usual.

Los postulados de $D$ (esquemas de axiomas y reglas primitivas de inferencia) son los siguientes:

a) Postulados del cálculo proposicional

$$
\begin{aligned}
& A \rightarrow(B \rightarrow A) \\
& (A \rightarrow B) \rightarrow((A \rightarrow(B \rightarrow C)) \rightarrow(A \rightarrow C)) \\
& (\neg A \rightarrow \neg B) \rightarrow((\neg B \rightarrow A) \rightarrow B) \\
& \frac{A, A \rightarrow B}{B}
\end{aligned}
$$

b) Postulados morales

$$
\begin{aligned}
& O_{m}(A \rightarrow B) \rightarrow\left(O_{m} A \rightarrow O_{m} B\right) \\
& O_{m} A \rightarrow P_{m} A\left(P_{m} A={ }_{d f} \neg O_{m} \neg A\right) \\
& \frac{A}{O_{m} A}
\end{aligned}
$$

c) Postulados jurídicos

$$
\begin{aligned}
& O_{j}(A \rightarrow B) \rightarrow\left(O_{j} A \rightarrow O_{j} B\right) \\
& O_{j} A \rightarrow P_{j} A\left(P_{j} A={ }_{d f} \neg O_{j} \neg A\right) \\
& \frac{A}{O_{j} A}
\end{aligned}
$$

d) Postulados mixtos

$$
\begin{aligned}
& O_{j} A \rightarrow O_{m} A \\
& O_{m} A \rightarrow P_{j} A
\end{aligned}
$$

Los conceptos de demostración y de teorema se definen de la manera acostumbrada. La noción de deducción impone que las reglas

$$
\frac{A}{O_{m} A} \mathbf{y} \frac{A}{O_{j} A}
$$


solamente se apliquen a teoremas y no puedan ser utilizadas en general en las deducciones, salvo cuando se aplican a teoremas. Así no se incurre en falacia naturalista violando el principio de Hume. La noción de consecuencia sintáctica queda caracterizada como en da Costa y Carnielli (1986).

Fácilmente se verifica que en el sistema $D$ vale el teorema de la deducción. Tenemos también:

Teorema 1: Los esquemas siguientes son teoremas en $D$ :

$$
\begin{array}{ll}
O_{m} A \rightarrow P_{j} A & V_{m} A \rightarrow \neg O_{j} A \\
V_{j} A \rightarrow V_{m} A & O_{j} A \rightarrow P_{m} A \\
O_{m}\left(O_{j} A \rightarrow O_{m} A\right) & \\
O_{j} A\left(O_{j} A \rightarrow O_{m} A\right), &
\end{array}
$$

donde $V_{j} A={ }_{d f} O_{j} \neg A$ y $V_{m} A={ }_{d f} O_{m} \neg A$.

Cabe formular una semántica para $D$ a partir de la noción de estructura- $D$, definida como sigue:

Una estructura $D$ es un cuádruplo $\left(W, R^{m}, R^{j}, \vdash\right)$, en la cual:

1) $W$ es un conjunto no vacío de elementos (conjuntos de mundos);

2) $R^{m} \subset W \mathrm{XW}$ y $\emptyset \neq R^{j} \subset R^{m}$ ( $R^{m}$ es una relación de accesibilidad moral y $R^{j}$ es una relación de accesibilidad jurídica);

3) Si $w \in W$, entonces existe un $w^{\prime} \in W$, tal que $w R^{m} w^{\prime}$;

4) Si $w \in W$ y $w$ pertenecen al dominio de $R^{j}$, entonces existe un $w^{\prime} \in W$, de modo que $w R^{j} w^{\prime} ; y$,

5) † es una relación de forzamiento entre mundos y fórmulas, que posee todas las propiedades usuales con referencia a los conectivos clásicos.

Cabe definir, sin dificultad, la noción de consecuencia semántica, representada por $1=$.

Teorema 2 (teorema de corrección y completitud): Sea $\Gamma \cup\{A\}$ un conjunto de fórmulas de $D$. Tenemos: 


$$
\Gamma \vdash A \Rightarrow \Gamma \vDash A \text {. }
$$

En $D$ obtenemos resultados como los siguientes:

$$
F_{m} A \rightarrow F_{j} A \text { y } V_{j} A \rightarrow V_{m} A,
$$

donde $F_{m} A={ }_{d f} P_{m} A \wedge P_{m} \neg A, \mathrm{y}$

$$
F_{j} A={ }_{d f} P_{j} A \wedge P_{j} \neg A .
$$

Vale decir, por lo tanto, que lo moralmente facultativo implica facultamiento jurídico, mientras que lo jurídicamente prohibido acarrea prohibición moral. Estas derivaciones llevan a cuestionar la adecuación de $D$ para formalizar ciertos discursos éticos. Obviamente, éste y otros resultados nos permiten comprender un poco mejor cuáles son las interrelaciones que se postulan entre modalidades morales y jurídicas, interrelaciones que dependen de la aceptación hipotética de los postulados de $D$.

Así, parece claro que hay cosas que pueden parecernos moralmente indiferentes o facultativas y que no por ello son jurídicamente facultativas. El ejemplo banal de las reglas de tránsito, donde el circular por la derecha es moralmente indiferente, aunque sea jurídicamente obligatorio, lo confirma, aunque cabría encontrar ejemplos en que la modalidad coincide en derecho y en moral. Pero, en general, los postulados mixtos de $D$ conducen a ciertas consecuencias contraintuitivas. Los propios axiomas del sistema parecen admitir, en ciertos casos, interpretaciones contraintuitivas. No es correcto que todo lo que sea jurídicamente obligatorio sea también moralmente obligatorio, como resulta del ejemplo clásico del deber jurídico de pagar impuestos que, según sostenía el Aquinate, no constituye obligación moral. Por contraposición tendríamos que reaparece la tesis de que aquello que está moralmente permitido omitir también lo estaría jurídicamente, tesis que los juristas sólo aceptarían con justificada alarma. Y, así, alcanzamos teoremas que 
suscitarían inquietud en el jurista tradicional y, suponemos, en el moralista teórico. Pero algunas de estas conclusiones, con todo, son presupuestos de tendencias expresas del pensamiento moral y jurídico. La proposición demostrable de que todo lo que estuviere moralmente prohibido implicaría la permisión jurídica de omitirlo es tesis que refleja rotundamente la pretensión clásica del iusnaturalismo religioso, pero que descartan no solamente las líneas principales teóricas del positivismo jurídico, sino también todo jurista dedicado a la labor dogmática. Igualmente, parece poco convincente tener que aceptar que lo jurídicamente obligatorio sea siempre moralmente permitido o, aún más, el teorema que declara que es moralmente obligatorio que aquello que es jurídicamente obligatorio obligue también moralmente —esto es: $\left(O_{m}\left(O_{j} A \rightarrow O_{m} A\right)\right)$. Estas consecuencias parecen constituir tesis propias de la grave actitud ideológica que N. Bobbio caracterizó como positivismo ideológico, que hace del imperativo contingente de un legislador una orden moralmente necesaria, como notoriamente lo establece la Epístola a los Romanos. Vernengo (1989) agrega a este respecto que "este teorema tiene su correlato en el enunciado no menos alarmante de que se está jurídicamente obligado a aceptar que todo lo que sea jurídicamente obligatorio es también moralmente obligatorio: $\left(O_{j}\left(O_{j} A \rightarrow O_{m} A\right)\right)$ "; estamos frente a un principio que pareciera contrario a la noción de autonomía moral o propio de una moral excesivamente legalista.

Advirtamos, para concluir esta revisión del sistema $D$, que el postulado $O_{m} A \rightarrow P_{j} A$ es superfluo en $D$. Y, en lugar del postulado $O_{j} A \rightarrow O_{m} A$, podemos asumir otro más débil, como, por ejemplo,

$$
O_{j} A \rightarrow P_{m} A
$$

cuyas consecuencias conviene explicar. Tendremos así un nuevo sistema, $D^{\prime}$, que pasamos a estudiar. 
2.2. El sistema $D^{\prime}$

Farmalinamne ahoma ntrn,cictama nmonncininnal $h^{\prime}$ tamhión los primitivos que $D$. En cuanto a los postulados, $D^{\prime}$ posee los mismos que $D$ en lo que hace a los incisos a, b y $c$ de la sección 2.1. Los postulados mixtos son, en cambio:

d) Postulados mixtos

$$
\begin{aligned}
& O_{j} A \rightarrow P_{m} A \\
& O_{m} A \rightarrow P_{j} A .
\end{aligned}
$$

Nuevamente aquí nos parece prima facie intuitivamente aceptable admitir que lo jurídicamente obligatorio deba estar moralmente permitido. Por lo menos, se trata de un principio que nos parece válido para un derecho ideal. El principio queda codificado en $D^{\prime}$ por el postulado indicado:

$$
O_{j} A \rightarrow P_{m} A \text {. }
$$

Resulta, por un lado, que este postulado puede ser probado en $D$. Lo mismo acontece con la fórmula

$$
O_{m} A \rightarrow P_{j} A,
$$

que es teorema tanto en $D$ como en $D^{\prime}$, no siendo necesario, pues, postularla como axioma.

Advirtamos también que si tales principios son aceptados (a saber: los postulados mixtos), valen también los siguientes:

$$
\begin{gathered}
V_{j} A \rightarrow \neg O_{m} A \\
V_{m} A \rightarrow \neg O_{j} A .
\end{gathered}
$$

En $D^{\prime}$ tenemos consecuencias semejantes al principio clásico ex falso quodlibet, en cuanto a fórmulas contradictorias o que implican contradicción deóntica, cabe derivar la permisión de cualquier acto. Por ejemplo el teorema 


$$
\left(V_{m} A \wedge O_{m} A\right) \rightarrow P_{j}(A \wedge \neg A),
$$

vale decir: si algo está moralmente prohibido y es moralmente obligatorio, está permitido jurídicamente tanto omitir el acto como llevarlo a cabo. Pero esa consecuencia define a los actos facultativos que, si se quiere, son los actos no regulados normativamente (pues un acto es facultativo cuando ni es obligatorio ni está prohibido). En mérito al teorema en cuestión la modalidad facultativa no sólo equivaldría a la conjunción de las negaciones de obligaciones y prohibiciones, sino también a la conjunción de obligaciones y prohibiciones, cosa que parece no corresponder a la comprensión normal de una acción libre. La noción de facultativa se convierte en paradójica en este sistema.

Nuevamente aquí observamos que, a partir de postulados prima facie razonables e intuitivamente satisfactorios, derivan consecuencias contraintuitivas o repudiables. Quizás sea que la estructura o estructuras lógicas que el derecho y la moral presuponen son más complicadas y delicadas de lo que se admite normalmente; ello no debe sorprender demasiado, pues aun en logica clásica, como es bien sabido, de principios intuitivamente aceptables derivan consecuencias parad6jicas, como las numerosas a que da lugar la implicación material. Nuestro postulado en este sistema no parece, en consecuencia, especialmente interesante. En cambio esquemas como

$$
\begin{gathered}
O_{m}\left(O_{j} A \rightarrow O_{m} A\right), \mathrm{y} \\
O_{j}\left(O_{j} A \rightarrow O_{m} A\right)
\end{gathered}
$$

no se obtienen en el sistema $D^{\prime}$, lo que parece razonable, coincidiendo con nuestras intuiciones, que hacen de ellos principios poco convincentes ( $c f r$. Vernengo, 1989).

Conviene, pues, efectuar algunas reflexiones sobre el significado de $D^{\prime}$. Es verdad, en efecto, que los postulados mixtos de este sistema también ofrecen ejemplos de consecuencias 
contraintuitivas. Hay casos conocidos en los cuales lo que es jurídicamente debido no es moralmente permitido: aś, en el ejemplo clásico, del deber del soldado de matar en guerra, frente a la prohibición moral general de matar. De este modo argumentan los conscience objectors y muchas sectas religiosas. Y tampoco todo lo que es moralmente obligatorio está siempre jurídicamente permitido. La institución del sutee en la India, por ejemplo, según la cual la mujer casada estaba obligada moralmente a inmolarse en la pira funeraria del marido, pese a que el derecho positivo colonial no se lo permitiera. 0 , para dar otro ejemplo, piénsese en el caso de la pobre Antígona, obligada moralmente a enterrar al hermano, pero a la que una disposición jurídica positiva emitida por otro hermano se lo prohibía jurídicamente. Esta situación fue siempre tomada como punto de partida para una reflexión sobre las relaciones entre el derecho y la moral, o bien, entre el derecho positivo y el derecho natural.

El primer postulado, $O_{j} A \rightarrow P_{m} A$, es la tesis clásica iusnaturalista de subalternación del derecho a la moral. El segundo, $O_{m} A \rightarrow P_{j} A$, expresa algo así como un principio kantiano: los deberes morales son parte o están incluidos en el dominio de la libertad, en el sentido de que todo lo que sea jurídicamente obligatorio debe ser moralmente posible. Sin embargo, la permisión jurídica débil, $P_{j}$, en el último postulado es, como sucede con la interpretación de la permisión en derecho, algo ambigua. Tenemos, pues, que en $D^{\prime}$ las nociones de permisión débil y fuerte (facultativa) crean problemas. Como se sabe, estas nociones son difícilmente interpretables en un plano puramente sintáctico.

De ahí que parezca que el principio clásico del iusnaturalismo escolástico: $V_{m} A \rightarrow \neg O_{j} A$ (no es jurídicamente obligatorio lo moralmente prohibido), que en una interpretación "débil" puede ser plausible (puedo dejar de hacer jurídicamente lo que la moral me prohíbe) en una interpretación "fuerte" no sería aceptado explícitamente por los juristas, puesto que 
significaría que quienquiera puede negarse a cumplir sus obligaciones jurídicas cuando ellas sean consideradas moralmente inaceptables por alguien. Se trata, en esencia, de la tesis anarquista clásica, toda vez que se considere a la moral como un código con validez autónoma subjetiva.

Intentemos, pues, examinar otra posibilidad formal: reemplazar el axioma $O_{j} A \rightarrow O_{m} A$ por otro aún más débil. Designaremos $D^{\prime \prime}$ el nuevo sistema así logrado, que pasamos a estudiar en la subsección siguiente.

\subsection{El sistema $D^{\prime \prime}$}

$D^{\prime \prime}$ posee los mismos símbolos primitivos de $D$. Inclusive, posee postulados idénticos a los de $D$, en lo que hace a los incisos a, b y c de la sección 2.1. En cambio, los postulados correspondientes al inciso $d$ son los siguientes:

d) Postulados mixtos

$$
\begin{aligned}
& P_{m} A \rightarrow P_{j} A \\
& O_{m} A \rightarrow P_{j} A
\end{aligned}
$$

Prácticamente todos los resultados de las secciones anteriores se extienden al sistema $D^{\prime \prime}$. En particular, cabe probar que los siguientes esquemas son válidos:

$$
\begin{array}{ll}
V_{m} A \rightarrow \neg O_{j} A & O_{j} A \rightarrow O_{m} A \\
O_{j} A \rightarrow P_{m} A & V_{j} A \rightarrow V_{m} A
\end{array}
$$

Nuevamente algunas de estas consecuencias parecen cuestionables, pues no parece aceptable que todo lo que sea jurídicamente obligatorio implique obligación moral ni, mucho menos, que las prohibiciones jurídicas, como la de transitar por la izquierda, impliquen interdicción moral. Ello da a nuestras derivaciones un sabor autoritario: los dictadores suelen pretender que lo que autoritariamente prescriben obligue moralmente a los súbditos, como dice el segundo de los esquemas indicados. Nuevamente, pues, conviene poner en claro que 
los postulados de $D^{\prime \prime}$ ofrecen dificultades para una interpretación intuitiva amplia, aunque sus postulados mixtos iniciales parezcan más bien aceptables. Con todo, el postulado mixto $P_{m} A \rightarrow P_{j} A$ expresa algo así como una legalización de lo que está moralmente permitido; su contraposición, $V_{j} A \rightarrow V_{m} A$, ciertamente sería rechazada en muchísimos casos. Piénsese en los movimientos que no admiten que la prohibición jurídica de ciertos actos, como la eutanasia, el aborto, o las actividades homosexuales, supongan condena moral en todos los casos. Justamente los defensores de tales cosas sustentan expresamente la tesis de que la prohibición legal de dichos actos no supone el repudio moral de los mismos. De otra suerte, la moral quedaría como parte del derecho y, por consiguiente, quedaría excluida también una crítica moral del derecho.

En la lógica deóntica normal se acepta que todo acto es obligatorio, prohibido o facultativo: $O A \vee V A \vee F A$ (así, por ejemplo, en von Wright, 1983; Alchourrón y Bulygin, 1974; Hilpinen, 1989; Soeteman, 1987 y Vernengo, 1976). Las acciones libres del sujeto, por lo menos desde un punto de vista jurídico, son aquellas que no está obligado a cumplir ni obligado a omitir, vale decir, las facultativas. Pero esta noción siempre ha dado problemas en la teoría ética y en la teoría general del derecho. Sin embargo, el moralista y el jurista consideran que sólo tiene sentido pensar acciones obligatorias o prohibidas en la medida en que el sujeto disponga de un dominio de acciones libres. La fórmula con la que concluimos esta sección expresa ese presupuesto; el sujeto debe ser, o es, libre para que pueda verse sujeto a obligaciones o prohibiciones. Esto es: se prohíbe, se obliga y, en consecuencia, se permite débilmente, porque se cree o presupone que el agente tiene libertad para decidir y para actuar. Esta suposición fundamental es algo así como una tesis de existencia: hay libertad en el campo del derecho y de la moral. En los sistemas $D, D^{\prime}$ y $D^{\prime \prime}$ supongamos que contamos con una proposición que expresa una acción o acto. La misma queda representada con la constante proposicional $K$. 
Entonces, el principio que consideramos, por lo menos en lo que se refiere a ese acto, se reduce a la fórmula $P K \wedge P \neg K$; vale decir, se reduce a afirmar que $K$ es facultativa $(F K)$. Por consiguiente, y como es obvio, en los sistemas estudiados no podemos probar la existencia de actos libres, pues la libertad jurídica no deriva de la lógica. No creemos, pues, conforme a una asentada tradición del pensamiento jurídico, que haya teorías válidas que sirvan para demostrar la existencia de actos libres. La existencia de los mismos es un dato contingente. En el terreno del simbolismo lógico, si hemos definido, como se suele hacer en la lógica normal, los operadores $O$ y $V$ (id.: $O \neg$ ) como contrarios, cabe también definir un tercer dominio funcional resultante de la negación de ambos, $\neg O A \wedge \neg V A$, que determina el operador $F$ utilizado en los sistemas anteriores. No parece admisible, en cambio, partir de $\boldsymbol{F}$ como operador primitivo (Paulson, 1988; Vernengo, 1989b), sin admitir supuestos metafísicos que la lógica no convalida. Que haya actos que satisfagan a ese operador $F$ es cosa contingente, aun cuando, por la manera utilizada para definir los operadores deónticos, su posibilidad lógica no pueda ser excluida. El tema, como lo reflejan los trabajos recientes, ofrece ambigüedades importantes, incluso en el nivel del simbolismo lógico (Opalek y Wolenski, 1973, 1986; Alchourrón y Bulygin, 1988). Veamos, pues, otros sistemas lógicos donde la existencia de ciertos hechos es tomada en cuenta.

Tanto en $D^{\prime}$ como en $D^{\prime \prime}$ cabe derivar un teorema del siguiente tenor:

$$
\left(O_{m} A \vee O_{j} A\right) \rightarrow\left(P_{m} A \leftrightarrow P_{j} A\right)
$$

Vale decir: si bien ambos sistemas permiten distinguir obligaciones y prohibiciones morales y jurídicas, en el dominio de lo permitido, moral y derecho son indiscernibles. Esta tesis tiene algún interés filosófico. Frente a las éticas tradicionales de impronta kantiana que consideran al deber (esto es: a la 
modalidad obligatoria y su correlato para las omisiones, la modalidad prohibitoria) como la modalidad primitiva y básica del pensamiento ético, algunos pensadores han propuesto considerar a la libertad (cuya modalidad deóntica se expresa con permisiones) como el dato ético primitivo. Obligaciones y permisiones son especificaciones limitativas de la libertad básica. Se trata, claro está, de tesis de naturaleza metafísica que quizás puedan ser analizadas con sistemas lógicos semejantes a $D^{\prime}$ y $D^{\prime \prime}$, donde no cabe diferenciar las permisiones jurídicas de las permisiones morales, pues lógicamente se coimplican en tanto demostrablemente equivalentes. La libertad, por decirlo así, es única; los deberes de hacer o de omitir, en cambio, pueden ser tanto morales como jurídicos.

\section{Lógica modal alético-deóntica}

Una lógica modal alético-deóntica es un sistema lógico que contiene no sólo modalidades dénticas (morales y jurídicas), sino también modalidades aléticas.

Una relación importante entre esos dos tipos de modalidades dénticas y aléticas está dada por el principio de Hintikka, $\square A \rightarrow O_{m} A$, valedecir: lo necesario implica obligación moral.

En esta sección estudiaremos un sistema proposicional de este tipo, que designaremos como $\bar{D}$, que tiene al cálculo proposicional clásico como lógica subyacente.

Los símbolos primitivos de $\bar{D}$ son los mismos que los de $D$, más el operador $\square$ (necesario).

Los postulados de $\bar{D}$ son los de los incisos a, b y c de $D$. Como postulados mixtos tenemos:

d) Postulados mixtos

$$
\begin{aligned}
O_{j} A & \rightarrow P_{m} A \\
\square A & \rightarrow O_{m} A
\end{aligned}
$$

Los postulados aléticos son los siguientes: 
e) Postulados aléticos

$$
\begin{aligned}
& \square(A \rightarrow B) \rightarrow(\square A \rightarrow \square B) \\
& \square A \rightarrow A \\
& \frac{A}{\square A}
\end{aligned}
$$

Fácilmente verificamos que los siguientes esquemas valen como teoremas:

$$
\begin{array}{ll}
\square A \rightarrow P_{m} A & O_{m} A \rightarrow \diamond A \\
\square A \rightarrow P_{j} A & O_{m} A \rightarrow P_{j} A \\
O_{j} A \rightarrow \diamond A & P_{m} A \rightarrow \diamond A
\end{array}
$$

Como consecuencia de los resultados antes mencionados, obtenemos las siguientes fórmulas:

$$
\begin{gathered}
O_{m} A \rightarrow \diamond A, \mathbf{y} \\
O_{j} A \rightarrow \diamond A,
\end{gathered}
$$

esto es: lo que es obligatorio, moral y jurídicamente, implica la posibilidad. Así pues, el axioma de Kant es válido. Y vale también el conocido principio jurídico de que lo imposible no obliga.

Para formular una semántica para $\bar{D}$, requerimos del concepto de estructura $\bar{D}$. Una $\bar{D}$-estructura es un quíntuplo (W, $\left.R^{m}, R^{j}, R^{\mathrm{D}}, \vdash^{-}\right)$, de tal suerte que:

1) $W$ es un conjunto no vacío de elementos.

2) $R^{m}, R^{\square} s \subset W \mathrm{XW}, \emptyset \neq R^{j} \subset R^{m}$, y $\emptyset \neq R^{j} \subset R^{\mathrm{a}}$.

3) Si $w \in W$, entonces existe $w^{\prime} \in W$, tal que $w R^{m} w^{\prime}$.

4) Si $w \in W$ y $w$ pertenecen al dominio de $R^{j}$, entonces existe $w^{\prime} \in W$, de modo que $w R^{i} w^{\prime}$;

5) $R^{\mathrm{a}}$ es reflexiva; y

6) t es una relación de forzamiento entre mundos y fórmulas que posee las propiedades usuales en lo que hace a los conectivos. 
Por medio de la noción de estructura $\bar{D}$, podemos introducir los conceptos semánticos básicos, en particular el de consecuencia semántica. Se prueba así que $\bar{D}$ es, en lo relativo a esa semántica, correcto y completo.

\section{Lógica deóntica no clásica}

Los cálculos proposicionales $D, D^{\prime}, D^{\prime \prime}$ y $\bar{D}$ constituyen sistemas clásicos, pues están basados en la lógica proposicional clásica. Dado que existen lógicas distintas de la lógica clásica, como las paraconsistentes y paracompletas, podríamos tomar una de esas lógicas como punto de partida para construir nuestra lógica deóntica. Obtendríamos así sistemas deónticos no clásicos. Creemos que vale la pena, por motivos que quedarán en claro más adelante, desarrollar una lógica jurídica no clásica. En esta sección, expondremos algunas razones para construir tales lógicas, en especial lógicas paraconsistentes.

Los códigos jurídicos y morales existentes contienen, en la mayoría de los casos, normas que acarrean contradicciones. De un corpus normativo - un código-, por ejemplo, puede derivarse que una acción es obligatoria y, al mismo tiempo, prohibida. Inclusive, en ciertas circunstancias puede darse que una acción está caracterizada como obligatoria y, simultáneamente, no serlo. Esto se produce, v. gr., en los llamados dilemas morales y, en general, con los dilemas deónticos (cfr. Puga, 1985 y Puga y da Costa, 1987c). De esta manera, si nuestra lógica subyacente fuera una lógica clásica, nos veríamos llevados, como se sabe, a una trivialización deóntica o a una trivialización propiamente dicha. En efecto, en nuestros sistemas usuales de lógica (jurídica o moral), de un dilema deóntico se deduce que cualquier acción es obligatoria, así como de una contradicción se deriva cualquier proposición. Luego, si utilizamos pura y simplemente la lógica clásica somos conducidos a trivialización. Para superar esas dificultades hay varios caminos posibles: 1) debilitar los postulados deónticos, tanto jurídicos 
como morales, manteniendo la lógica clásica como lógica subyacente; 2) recurrir a una lógica paraconsistente; 3) proceder al modo de Alchourrón, Makinson y otros autores (para los detalles, cfr. Weinberger, 1975 y los ensayos de Alchourrón y Makinson sobre el tema en Hilpinen, 1981 ; cfr. también Miró Quesada, 1988), quienes procuran sistematizar la eliminación de inconsistencias en sistemas normativos, recuperando la consistencia mediante recursos formales; 4) substituir la noción clásica monotónica de consecuencia por lógicas no monotónicas.

Como señalamos, una cuestión importante, que nos lleva a una lógica deóntica paraconsistente, es la de los dilemas morales ( $c f r$. R. Routley y V. Plumwood, 1984). Una persona enfrenta un dilema moral cuando para ella es obligatorio efectuar una acción, pero al mismo tiempo también le es obligatorio no llevarla a cabo. Un ejemplo simple de dilema moral lo constituye el aborto. Supongamos que un hombre, en el hospital donde su mujer va a dar a luz, es informado por los médicos que debe decidir si se salva la vida de la madre o la del hijo. En todo caso, uno de los dos deberá morir. Esta situación coloca a la persona en un dilema normativo ( $c f r$. da Costa y Carnielli, 1986). En general, un dilema normativo se expresa en un conflicto normativo frente al cual el razonamiento jurídico o moral no tiene pautas lógicas convincentes (Hilpinen, 1985).

Otro problema, frente al cual las lógicas clásicas no son enteramente satisfactorias proviene del tradicional problema de las lagunas en el derecho y de la circunstancia notoria de que muchos conceptos morales y jurídicos son algo vagos. Para problemas de este tipo se han propuesto lógicas divergentes diferentes. Una categoría de lógica que se ha sugerido utilizar en el terreno del discurso ético es la de lógicas fuzzy o naturales, que permitirían razonar en contextos conceptuales difusos o incompletos. Estas lógicas destinadas a hacer frente a la vaguedad llevan, en ciertas circunstancias, no sólo a inconsistencias (contradicciones), sino, además, son paracompletas (habrá 
algunas acciones no encuadradas en las normas del código). Dado que los corpus jurídicos normalmente presentan lagunas, un modo de abordar lógicamente el problema podría ser mediante algunas de esas lógicas (cfr. sobre el punto, Alchourrón y Bulygin, 1974 y von Wright, 1989).

Pero hay otras razones para aplicar lógicas no clásicas en el terreno de la lógica dé́ntica. Con todo, no discutiremos este tema aquí. Nos limitaremos, en buena parte de lo que sigue, a presentar algunos sistemas paraconsistentes dénticos que permitan manejar códigos normativos con contradicciones, en los cuales los dilemas éticos (morales o jurídicos) no queden excluidos a priori. A este respecto, tenemos presente la sugerencia de Hilbert que hemos citado como encabezado de este ensayo: se refiere a la axiomatización de la física y afirma que interesa téricamente elaborar sistemas formales que sirvan para reconstruir teorías posibles como variaciones, quizás, de otras teorías aceptadas por razones intuitivas informales. Puede ser, pensamos, que las variaciones introducidas en los sistemas lógicos deónticos subyacentes, explícita o implícitamente asumidos por moralistas y juristas, permitan una mejor comprensión crítica de las teorías que ellos proponen y de sus consecuencias derivables.

\subsection{Logica deóntica bidimensional paraconsistente}

Si en lugar de adoptar el cálculo proposicional clásico como lógica subyacente, tomáramos el cálculo paraconsistente $C$, (cfr. da Costa, 1974), obtendríamos un sistema de lógica deóntica paraconsistente con modalidades morales y jurídicas.

Tal sistema, designado como $D_{1}$, tiene los siguientes símbolos primitivos: 1) conectivos: $\neg$ (negación), $\rightarrow$ (implicación), $\vee$ (disyunción), $\wedge$ (conjunción), $O_{m}$ (obligatorio moralmente), y $O_{j}$ (obligatorio jurídicamente); 2 ) variables proposicionales; y 3 ) paréntesis. El símbolo $\leftrightarrow$ (equivalencia) es definido como 
en lógica clásica, y los conceptos de fórmula, demostración y teorema son los usuales.

Los postulados de $D_{1}$ son los siguientes:

a) Postulados de $C_{1}$ :

$$
\begin{aligned}
& A \rightarrow(B \rightarrow A) \\
& (A \rightarrow B) \rightarrow((A \rightarrow(B \rightarrow C)) \rightarrow(A \rightarrow C)) \\
& \frac{A, A \rightarrow B}{B} \\
& (A \wedge B) \rightarrow A \\
& (A \wedge B) \rightarrow B \\
& A \rightarrow(B \rightarrow(A \wedge B)) \\
& A \rightarrow(A \vee B) \\
& B \rightarrow(A \vee B) \\
& (A \rightarrow C) \rightarrow((B \rightarrow C) \rightarrow((A \vee B) \rightarrow C)) \\
& A \vee \neg A \\
& \neg \neg A \rightarrow A \\
& B^{\circ} \rightarrow((A \rightarrow B) \rightarrow((A \rightarrow \neg B) \rightarrow \neg A)) \\
& A^{\circ} \wedge B^{\circ} \rightarrow\left((A \wedge B)^{\circ} \wedge(A \rightarrow B)^{\circ} \rightarrow(A \vee B)^{\circ}\right)
\end{aligned}
$$

b) Postulados morales:

$$
\begin{aligned}
& O_{m}(A \rightarrow B) \rightarrow\left(O_{m} A \rightarrow O_{m} B\right) \\
& O_{m} A \rightarrow P_{m} A \\
& \frac{A}{O_{m} A} \\
& A^{\circ} \rightarrow\left(O_{m} A\right)^{\circ}
\end{aligned}
$$

c) Postulados jurídicos:

$$
\begin{aligned}
& O_{j}(A \rightarrow B) \rightarrow\left(O_{j} A \rightarrow O_{j} B\right) \\
& O_{j} A \rightarrow P_{j} A \\
& \frac{A}{O_{j} A} \\
& A^{\circ} \rightarrow\left(O_{j} A\right)^{\circ} \\
& \text { d) Postulados mixtos: }
\end{aligned}
$$


$O_{j} A \rightarrow O_{m} A$

$O_{m} A \rightarrow P_{j} A$.

Para estas fórmulas valen las siguientes definiciones:

$$
\begin{array}{ll}
A^{\circ}=_{d f} & \neg(A \wedge \neg A) \\
\neg \star A=_{d f} & \neg A \wedge A^{\circ} \\
P_{m} A==_{d f} & n e g \star O_{m} \neg \star A \\
P_{j} A={ }_{d f} & \neg \star O_{j} \neg \star A
\end{array}
$$

Los siguientes teoremas son fácilmente probados:

Teorema 1 (deducción): $\Gamma, A \vdash B \Rightarrow \Gamma \vdash A \rightarrow B$.

Teorema 2: Los siguientes son esquemas de teoremas en $D_{1}$ :

$$
\begin{aligned}
& O_{m} \leftrightarrow \neg \star P \neg \star A \\
& O_{j} \leftrightarrow \neg \star P_{j} \neg \star A \\
& A^{\circ} \rightarrow \neg\left(O_{m} A \wedge O_{m} \neg A\right) \\
& A^{\circ} \rightarrow \neg\left(O_{j} A \wedge O_{j} \neg A\right) \\
& \neg \star\left(O_{m} A \wedge \neg \star O_{m} A\right) \\
& \neg \star\left(O_{j} A \wedge \neg \star O_{j} A\right) \\
& \left(O_{m} \neg A \wedge A^{\circ}\right) \rightarrow O_{m} A \\
& \left(O_{j} \neg A \wedge A^{\circ}\right) \rightarrow O_{j} A \\
& \left(O_{m} \wedge O_{m} \neg \star A\right) \rightarrow O_{m} B \\
& \left(O_{j} \wedge O_{j} \neg \star A\right) \rightarrow O_{j} B \\
& \left(O_{m} A \rightarrow O_{m} B\right) \rightarrow\left(\neg O_{m} A \vee O_{m} B\right)
\end{aligned}
$$

Teorema 3: Los siguientes esquemas no valen en $D_{1}$ :

$$
\begin{aligned}
& (A \wedge \neg A) \rightarrow B \\
& O_{m} \neg A \rightarrow \neg O_{m} A \\
& O_{j} \neg A \leftrightarrow \neg P_{j} A \\
& \neg(A \wedge \neg A) \\
& O_{j} \neg A \rightarrow \neg O_{j} A \\
& (\neg A \wedge \neg B) \rightarrow \neg(A \vee B)
\end{aligned}
$$




$$
\begin{aligned}
& O_{m} \neg(A \wedge \neg A) \\
& \neg\left(O_{m} \neg A \wedge \neg O_{m} \neg A\right) \\
& (\neg A \vee \neg B) \rightarrow \neg(A \wedge B) \\
& O_{j} \neg(A \wedge \neg A) \\
& \neg\left(O_{j} \neg A \wedge \neg O_{j} \neg A\right) \\
& \left(O_{m} \wedge O_{m} \neg A\right) \rightarrow B \\
& A \leftrightarrow \neg \neg A \\
& O_{m} \neg A \leftrightarrow \neg P_{m} A \\
& \left(O_{j} A \wedge O_{j} \neg A\right) \rightarrow B
\end{aligned}
$$

Para otros detalles del sistema $D_{1}$, el lector puede remitirse a da Costa y Carnielli (1986). Sin embargo, conviene dejar en claro que la negación $\neg \star$ es una negación fuerte, que satisface todas las condiciones de la negación clásica. Por ello es conveniente definir los operadores $P_{m}$ y $P_{j}$ a partir de $O_{m}$ y $O_{j}$ y de dicha negación; de este modo, los operadores así definidos poseerán muchas de las propiedades clásicas. Además, cabría también definir otros operadores análogos con la negación débil $\neg$. Para fijar algunas ideas interpretaremos la definición

$$
P_{m} A={ }_{d f} \neg \star O_{m} \neg \star A
$$

en el sentido de que significa, como en la situación clásica, que es falso que sea moralmente obligatorio llevar a cabo la negación (clásica) de $A$. El postulado moral $A^{\circ} \rightarrow\left(O_{m} A\right)^{\circ}$, tendría que ser interpretado, a su vez, en el sentido de que si $A$ satisface las leyes de la lógica clásica, si funciona bien en ese contexto, entonces también $O_{m} A$ funciona correctamente.

El sistema $D_{1}$ permite un tratamiento lógico adecuado de los dilemas dénticos, dado que de una fórmula como

$$
O A \wedge O \neg A
$$

no se deduce que todo sea obligatorio y, de modo similar, de una contradicción, $B \wedge \neg B$, no cabe deducir cualquier fórmula. 
En otras palabras: los dilemas y las contradicciones no quedan excluidas $a b$ initio.

Así como construimos $D_{1}$, podemos construir nuevos sistemas deónticos bidimensionales paraconsistentes, como $D_{1}^{\prime}$ y $D_{1}^{\prime \prime}$. Basta reemplazar la lógica subyacente de $D$ y $D^{\prime \prime}$ por la lógica paraconsistente $C_{1}$. En los cálculos $D_{1}, D_{1}^{\prime}$ y $D_{1}^{\prime \prime}$ es posible encarar los dilemas éticos y ciertos tipos de contradicciones normativas sin correr el riesgo inmediato de una trivialización simple o deóntica.

Como en los sistemas paraconsistentes recurrimos a dos negaciones, $\neg$ y $\neg \star$, en donde la primera es paraconsistente y la segunda, clásica, fácilmente se entienden ciertas características de esos cálculos, como, por ejemplo, el hecho de no ser válidos en $D_{1}$ esquemas como $\left(O_{m} \neg A \rightarrow \neg P_{m} A\right)$, o bien, $\left(O_{j} A \wedge O_{j} \neg A\right) \rightarrow O_{j} B$. Sin embargo, si en lugar de la negación débil utilizáramos la negación fuerte, tales esquemas sí serían válidos. Cabría, también introducir operadores deónticos débiles, definidos a partir de la negación débil. No entraremos en este tema, pues aquí sólo nos interesa determinar las características generales de estas lógicas.

\subsection{Lógica modal alético-déntica paraconsistente}

Para elaborar un sistema proposicional paraconsistente, con modalidades dénticas y aléticas, reemplazaremos en $\tilde{D}$, el cálculo proposicional clásico por el cálculo $C_{1}$ de da Costa (1974). Este sistema, que denominaremos $\bar{D}_{1}$, tiene los mismos símbolos primitivos que $D_{1}$, más el símbolo $\square$.

Los postulados de $\bar{D}_{1}$ son los siguientes:

a) Postulados de $C_{1}$ : los mismos de la sección 4.1.

b) Postulados morales: los mismos de la sección 4.1.

c) Postulados jurídicos: los mismos de la sección 4.1.

d) Postulados aléticos: los mismos de la sección 3.

e) Postulados mixtos: 


$$
\begin{aligned}
& A^{\circ} \rightarrow(\square A)^{\circ} \wedge\left(O_{j} A\right)^{\circ} \wedge\left(O_{m} A\right)^{\circ} \\
& O_{j} A \rightarrow O_{m} A \\
& \square A \rightarrow O_{m} A
\end{aligned}
$$

Los dos últimos sistemas exhiben postulados mixtos que permiten inferir obligaciones morales de obligaciones jurídicas, circunstancia que, como se ha señalado, tiene un saber legalista y autoritario que la mayoría de los juristas no admitiría. Inclusive hay normas positivas, como en la Constitución argentina de 1853, que prohíben jurídicamente al juez extraer un deber moral de una mera obligación jurídica. Pero, por otro lado, es claro que hay líneas de pensamiento que refrendarían principios tan severos. En todo caso, la implicación conversa, que haría de todo deber moral una obligación jurídica, no vale en estos sistemas.

A partir de las ideas expuestas anteriormente, en la sección 3 , podemos generalizar la noción de estructura $\bar{D}$, construyendo una semántica correcta y completa para $\bar{D}_{1}$. Como $C_{1}$ es el primer cálculo de una jerarquía $C_{n}, 1 \leq n \leq w$ ( $c f r$. da Costa, 1974), podemos aun formalizar otras lógicas paraconsistentes con modalidades deónticas, tanto morales como jurídicas, así como con modalidades aléticas.

En el desarrollo integral de una lógica deóntica, de tipo moral o jurídico, se hace imprescindible considerar el problema de las modalidades iteradas, lo que investigaremos en trabajos futuros. Además, nos parece también necesario que, en lugar de operadores deónticos absolutos, tratemos de operadores relativizados (condicionados) a situaciones determinadas, expresadas mediante los enunciados adecuados. Los cálculos estudiados en este trabajo pueden ser extendidos a la lógica de primer orden y aun de órdenes superiores ( $c f r$, por ejemplo, Puga, 1985). Hasta aquí hemos tratado apenas de algunos sistemas posibles que tienen interés para nuestras investigaciones, sean puramente formales o bien materiales, en el campo 
de la ética. Algunas de las ideas aquí esbozadas podrán ser desarrolladas más adelante.

\section{Consideraciones finales}

No se pretende, mediante formalización, condensar en unas formulas toda la riqueza de concepciones informales existentes. Por el contrario, como se sugirió inicialmente, el objetivo de la formalización reside fundamentalmente en la construcción de sistemas que nos ayuden a comprender mejor aquellas concepciones informales, poniendo en claro, en particular, las presuposiciones implícitas y las dificultades encubiertas. Sólo mediante la formalización tomamos conciencia de la existencia de paradojas y de resultados contraintuitivos que el discurso no formalizado oculta. Contribuye, así, la formalización ensayada a una codificación total y explícita de la estructura lógica de discursos informales, como el jurídico y el moral, contribuyendo a un conocimiento mejor de nuestras teorías al respecto. Sin esta contrapartida formal, no tendríamos una ciencia propiamente dicha, armónica y sistemática. En suma, una formalización bien aplicada resulta ser extremadamente importante en la investigación detallada de la actividad científica, en sentido amplio, y de los contextos racionales correspondientes.

Creemos, pues, que nuestra exposición hace patente el poder de los métodos formales en el campo de la lógica deóntica y de las relaciones entre moral y derecho. En concreto, nos hizo prestar atención a ciertas paradojas o consecuencias contraintuitivas que surgen cuando se desea establecer relaciones funcionales entre esos dos dominios. Parece improbable que se llegue a una solución del problema mediante esquematizaciones simplistas; en este asunto, nuestro trabajo constituye solamente un paso inicial, dado que no abrigamos la ilusión de haber alcanzado soluciones definitivas. En el futuro se deberá recurrir, como advertimos, a técnicas más refinadas como, por ejemplo, lógicas con modalidades de naturaleza relativa, tanto 
morales como jurídicas. Con todo, los sistemas lógicos formales aquí propuestos para dar cuenta, aunque de manera parcial y provisoria, de ciertas teorías y concepciones nos ayudarán seguramente a comprenderlas mejor. Nuestro método consiste en algo así como elaborar aproximaciones sucesivas, como sucede, v. gr., en la búsqueda de soluciones a los problemas de la física y otras ciencias empíricas.

\section{BIBLIOGRAFIA}

Alchourrón, C., 1969, "Logic of Norms and Logic of Normative Proposition", Logique et Analyse, no. 12.

- y E. Bulygin, 1974, Introducción a la metodología de las ciencias jurídicas , Astrea, Buenos Aires (trad. inglesa, Normative Systems Springer Verlag, 1973).

- 1988, "Perils of Level Confusion in Normative Discourse", Rechtstheorie, vol. XIX, p. 230 ss.

— y D. Makinson, 1981, en Hilpinen (ed.), "Hierarchies of Regulations and Their Logic".

—y A.A. Martino, 1988, "Lógica sin verdad", en Theoria, San Sebastián, (versión italiana, "Logica senza verità", en Expert Systems in the Law, Bolonia, 1988).

Allen, L.E. y C.S. Saxon, 1986, "Analysis of the Logical Structure of Legal Rules by a Modernized and Formalized Version of Hohfeld Fundamental Legal Conceptions", en Automated Analysis of Legal Texts, North-Holland, p. 385 ss.

Bobbio, N., 1979, Il positivismo giuridico, Giappichelli, Turín.

Bulygin, E., 1986, "Permissive Norms and Normative Systems", en Automated Analysis of the Law, Reidel, p. 211 ss.

Cossio, C., 1945, La teoría egológica del derecho y el concepto jurídico de libertad, Buenos Aires.

da Costa, N.C.A., 1974, "On the Theory of Inconsistent Formal Systems", Notre Dame Journal of Formal Logic, no. 11, p. 497 ss. -y W.A. Carnielli, 1985, "On Paraconsistent Deontic Logic", Philosophia, no. 16, p. 293 ss.

Hilbert, D., 1900, “Mathematical Problems", en F.E. Browder (ed.), 
Mathematical Developments Arising from Hilbert Problems, American Mathematical Society, 1976.

Hilpinen, R. (ed.), 1971, Deontic Logic: Introductory and Systematic Readings, Reidel.

1981, New Studies in Deontic Logic, Reidel.

- 1985, "Normative Conflicts and Legal Reasoning", en Man, Law and Modern Forms of Life, Reidel.

$\longrightarrow$, 1989, “On Deontic Logic, Pragmatics and Modality", en H. Stachiwiak (ed.), Pragmatics.

Hintikka, J., 1985, “Legal Reasoning and Legal Systems", en Man, Law and Modern Forms of Life, Reidel.

Kelsen, H., 1960, Reine Rechtslehre, 2a. ed., Viena.

McCarty, L.T., 1986, "Permissions and Obligations: An Informal Introduction", en Automated Analysis of the Law, Reidel, p. 307 ss. Miró Quesada, F., 1975, Ensayos de filosofía del derecho, Lima.

—_, 1988, “Lógica jurídica idiomática”, en João Pessoa (ed.), Actas III, Congresso Brasileiro de Filosofia do Direito.

Opalek, K. y J. Wolensky, 1973, “On Weak and Strong Permission”, Rechtstheorie, vol. IV.

$\longrightarrow, 1986$, "On Weak and Strong Permission Once More", Rechtstheorie, vol. XVlI.

Paulson, S.L., 1988, “An Empowerment Theory of Legal Norms", Ratio Juris, Oxford, no. 1, p. 58 ss.

Puga, L.Z., 1985, Uma lógica do querer, P. Universidade Católica de São Paulo, São Paulo.

—_, 1988, "A lógica deôntica e a teoria tridimensional do direito", Revista de Tribunais, São Paulo, no. 634.

- y da Costa, N.C.A., 1987a, "Logic With Deontic and Legal Modalities", Bulletin Sec. Logic Polish Academy of Sciences, no. 2, p. 71 ss.

—, 1987b, "Lógica deôntica e direito", Boletim da Sociedade Paranaense de Matemática, no. 2, p. 141 ss.

,$- 1987 \mathrm{c}$, “Sobre a lógica deôntica não clássica”, Crútica, UNAM, México, vol. XIX, no. 55, p. 19 ss.

—y R.J. Vernengo, 1989, "Lógica, ética e direito", ponencia presentada en el III Simpósio Latino Americano de Lógica Matemática, Paraíba, 1989. Próximamente aparecerá publicada en la revista $U$. de Campinas.

Reale, M., 1986, Teoria tridimensional do direito, Saravia, São Paulo. 
Routley, R. y V. Plumwood, 1984, "Moral Dilemmas and Logic of Deontic Notions", RSSS, The Australian National University.

Soeteman, A., 1987, "A Weak and Strong Permission in the Law", en A. Frändberg (ed.), The Stucture of Law, Uppsala.

Vernengo, R. J., 1976, Curso de teoría general del derecho, 2a. ed., Buenos Aires.

—, 1987, “Derecho y lógica”, en Anuario de Filosofía del Derecho, Madrid.

—_, 1989a, "Moral y derecho: sus relaciones lógicas", Revista Jurídica de Buenos Aires, 1989-1, Buenos Aires.

__, 1989b, "About an Empowerment Theory of Legal Norms and Some Related Problems", Ratio Juris, Oxford, vol. II, no. 3.

$\longrightarrow$, 1990, "Dimensiones del derecho", en Homenaje a M. Reale, São Paulo.

von Wright, G.H., 1951, “Deontic Logic”, en Logical Studies, Routledge \& K. Paul.

- 1963, Norm and Action, Oxford.

—, 1983, "Norms, Truth and Logic", en Practical Reason: Philosophical Papers, vol. I, no. B, Blackwell.

- 1986, "Truth, Negation and Contradiction", Synthèse, no. 66, p. 3 ss.

_ 1989, "Logiche della verità", Informatica e Diritto, Florencia, vol. XV, (versión inglesa, "Truth Logics").

Weinberger, $0 ., 1975$, "Ex falso quodlibet in der deskriptiven und in der präskriptiven Sprache", Rechtstheorie, no. 6, pp. 17-22.

_ 1982, "Kelsens These von der Unadwendbarkeit logischer Regeln auf Normen", en Die Reine Rechtslehre in wissenschaftlicher Diskassion, Viena. 


\section{SUMMARY}

The relationships between moral and legal codes, that is, between specific normative sets, is a traditional problem of ethics, politics and law philosophy. Deontic logics have provided some instruments for a deeper analysis of those relations. The authors try to analyze the logical consequences of the adoption of different axiomatical logical systems, where deontic modalities are introduced as expressing different senses of moral or legal obligation, prohibition, permission, etc. It turns out, as a result of the analysis, that some very traditional thesis about the relationships between law and moral presuppose different logical requirements, some of which are not only interesting from a pure formal point of view, but also as an essay in a rational analysis of normative discourse. Different types of logics, specially some called bidimensional deontic systems, are investigated, as some mixed alethic-deontic and paraconsistent systems are sketched. Contraintuitive consequences and paradoxes deriving from those logical presuppositions are described and their consequences in ethical thinking are underlined.

The paper's purpose is to attain a better understanding of some informal ethical notions through the construction of formal systems expressing with some precision the very vague notions that sometimes are behind accepted legal or moral conceptions. Thus, the authors think, it would be possible a progressive aproximation to a better analysis of some important presuppositions of ethical discourse, a discourse where morals and law have an essential part to play.

[Roberto J. Vernengo] 